\title{
Política y Sociedad
}

ISSN: $1130-8001$

ISSN-e: $1988-3129$

\section{El franquismo a través de las urnas. Metodología, fuentes y retos para una aproximación electoral al régimen de Franco}

\author{
Carlos Domper Lasús ${ }^{1}$
}

Recibido: 20-12-2016/ Aceptado: 23-10-2017

Resumen. Este artículo pretende plantear una nueva perspectiva historiográfica desde la que aproximarse al estudio del franquismo, utilizando el análisis de las elecciones a concejales de representación familiar y a procuradores en Cortes en representación de las familias. Para ello, se combinan tanto la metodología histórica como la procedente de las ciencias sociales, aunque priorizando la primera de ellas, estableciéndose los criterios a seguir para definir adecuadamente el sujeto de investigación y para profundizar en él posteriormente. Por lo tanto, el artículo define un campo de investigación hasta ahora escasamente transitado en el estudio de la dictadura franquista, conectando investigaciones y bibliografía procedentes tanto de la historiografía como de la ciencia política.

Palabras clave: franquismo; apoyos sociales; Guerra Fría; elecciones; dictaduras; historia electoral; cooptación de élites; institucionalización.

\section{[en] Francoism through the Polls. Methodology, Sources and Challenges for an Electoral Approach to Franco's Regime}

\begin{abstract}
This article proposes a new perspective to approach the study of the Francoism by using the analysis of the elections to select both the councillors who represented the heads of families at the city councils and the members of the Spanish Cortes who represented the families. To do this, the paper combines both historical methodology and social science one, although prioritizing the first of them, establishing the criteria to be followed to properly define the subject of research and to deepen it. Therefore, the article defines a field of research hitherto little researched by Francoist dictatorship literature and brings together both research and bibliography coming from the historiography as well as the political science.
\end{abstract}

Keywords: Francoism; social support; Cold War; elections; dictatorships; electoral history; elites cooptation; institutionalization.

Cómo citar: Domper Lasús, C. (2018): "El franquismo a través de las urnas. Metodología, fuentes y retos para una aproximación electoral al régimen de Franco", Política y Sociedad, 55(1), pp. 115-134.

Sumario. 1. Introducción. 2. Una aproximación pluridisciplinar a un objeto multifacético. 3. Fuentes para una aproximación electoral al franquismo. 4. Algunos retos en torno al estudio de las elecciones, el fascismo español y la Guerra Fría. 5. Bibliografía.

Libera Università Internazionale degli Studi Socali ‘Guido Carli’ (Italia).

E-mail: carlosdomper82@gmail.com 
Agradecimientos. El autor forma parte del proyecto de investigación MICINN HAR2014-53498-P “Culturas políticas, movilización y violencia en España, 1930-1950".

\section{Introducción}

El desarrollo de enfoques novedosos para aproximarse al análisis del franquismo debe remitirnos, en primer lugar, a la ya dilatada historia de sus estudios, puesto que resulta harto difícil (re)pensar cualquier tema sin conocer las fortalezas y debilidades tanto de las principales visiones existentes sobre él, como de las líneas de investigación en curso. Afortunadamente esa labor ha sido realizada ya en otros lugares por autores de contrastada solvencia (Sanz, 2013; Thomàs, 2008; Ortega, 2006; Rodríguez, 2006), por lo que nos limitaremos aquí a subrayar algunas ideas puestas de manifiesto en esos trabajos que nos permitan armar el punto de arranque de este artículo.

A día de hoy, la historiografía sobre el franquismo cuenta con una amplia trayectoria iniciada a principios de los años sesenta, cuando Stanley G. Payne comenzó a analizar aquello que él mismo denominó como "the Spanish fascism" (Payne, 1961). Al margen de los detalles concretos, los estudios sobre el tema han recorrido muy diversas etapas con una relación cambiante con respecto a los estudios internacionales sobre el fascismo que se ha reflejado en el vivo debate sobre la 'naturaleza política' del régimen (Pérez, 1994; Moradiellos, 2003; Saz, 2004; Rodrigo, 2009). No obstante, existe un consenso claro en torno a la idea de que los años noventa constituyeron un punto de inflexión en la evolución de los estudios sobre este periodo de la historia de España.

Tal y como han subrayado diferentes autores, durante aquélla década se produjo una importante expansión de este campo historiográfico caracterizada por un incremento de la producción, una creciente pluralidad temática, la renovación de los enfoques, el afinamiento de los instrumentos conceptuales y metodológicos, en muchas ocasiones incorporándolos desde otras disciplinas académicas, y la influencia de los estudios internacionales sobre el fascismo, que en los años noventa experimentaron también una importante renovación de signo tanto culturalista como social, paralela al resurgir de la comparación y del fascismo genérico (Sanz, 2013; Ortega, 2006).

A la luz de lo anterior, no cabe duda de que, como ha indicado Julián Sanz, la historiografía sobre el franquismo ha dado un salto cualitativo hacia adelante que ha conllevado la modificación de nuestra forma de entender la historia del régimen de Franco (Sanz, 2013: 57). No obstante, actualmente todavía es posible identificar en nuestro campo de estudio debilidades que en algunos casos han sido subrayadas repetidamente en los últimos veinte años y en otros ni tan si quiera han sido consideradas como un aspecto en cuya investigación merezca la pena invertir tiempo y esfuerzo. Entre ellas, y por lo que a este trabajo concierne, debemos destacar las siguientes. Por un lado, aunque en mejor situación que hace unos años, el desequilibrio persistente entre los trabajos dedicados al estudio del primer y del segundo franquismo, con el predominio de los dedicados al primero de ellos. En segundo lugar, conocemos todavía muy poco sobre el lugar de la dictadura en el marco comparado a partir de los años sesenta. Finalmente, la ausencia casi total de 
líneas de investigación relacionadas con los procesos electorales puestos en marcha por el franquismo, así como sobre sus procedimientos de representación política.

A decir verdad, ni las elecciones ni los plebiscitos organizados por las dictaduras europeas del siglo XX han sido nunca un tema relevante para la investigación histórica. Al considerar que la ausencia de lo que los politólogos denominan "competición política" (Altman, 2011; Roemer, 2001; Bogdanor, 1987; Sartori, 1976) convertía aquellos procesos electorales en simples eventos propagandísticos, los historiadores han priorizado el análisis de los discursos, las policías secretas o la violencia (entre otros muchos) como elementos más relevantes para explicar los mecanismos a través de los cuales los citados regímenes trataron de salvaguardar el control del poder.

No obstante, entre los escasos estudios existentes al respecto en el ámbito internacional existe una llamativa división asimétrica del interés académico en estas votaciones que ha provocado la presencia de diferentes aproximaciones, métodos y fuentes a la hora de investigarlos. Así, los historiadores han estudiado las elecciones y plebiscitos celebrados en la Italia fascista y la Alemania nazi, un hecho explicable por la posibilidad de acceder a las fuentes archivísticas desde el final de la Segunda Guerra Mundial (Dal Lago, 1999; Fimiani, 1997; Mühlen, 1979; Jung, 1995; Chrystal, 1975). Por su parte, los politólogos analizaron los procesos electorales que tuvieron lugar tanto en la URSS como en sus satélites del Este, sin acceso a documentación de archivo hasta 1989 y por tanto basándose en informaciones periodísticas, entrevistas a exiliados en los EE.UU. y las experiencias personales de los propios investigadores en sus estancias en la URSS (Swearer, 1961; Gilison, 1968; Friedgut, 1979). Por desgracia, después de 1991 los científicos sociales perdieron interés en las elecciones que se celebraron bajo las dictaduras comunistas para centrarse en investigar aquellas celebradas al calor de los nuevos regímenes surgidos de la desintegración de la Unión Soviética, lo que ha derivado en una de las corrientes de estudios más candentes de la ciencia política en la actualidad, el electoral authoritarianism (Schedler, 2006; Morse, 2012).

Esta dicotomía está también presente en los pocos trabajos existentes sobre las elecciones en la España de Franco. Dicha bibliografía se encuentra claramente dividida en dos bloques. Los primeros estudios realizados en España sobre este asunto aparecieron a mediados de los años setenta y fueron realizados por politólogos ligados al profesor Manuel Martínez Cuadrado alrededor de la celebración en París de un congreso sobre "elecciones no competitivas" (Martínez, 1974; Ruiz de Azúa, 1977; López, 1978; Vanaclocha, 1977). El título del congreso fue "Elecciones no-competitivas, semi-competitivas y la transición a los sistemas pluralistas competitivos", organizado en mayo de 1976 por el Seminario de Derecho Político de la Facultad de Ciencias Políticas de la Universidad Complutense de Madrid, la Fundación Juan March y la Foundation Nationale des Sciences Politiques de Paris. Las principales ponencias del mismo fueron publicadas en (Linz et al., 1978). Estos trabajos estuvieron muy influenciados por los resultados de las investigaciones previas realizadas por los politólogos sobre las elecciones en los regímenes comunistas bajo el manto del debate en torno al totalitarismo y el autoritarismo. 
Algunas décadas más tarde, en los años noventa, los historiadores Roque Moreno Fonseret y Francisco Sevillano Calero recuperaron el tema partiendo de los trabajos realizados en los años setenta, pero incorporando algunos de los resultados de la investigación histórica realizada sobre las elecciones en la Italia de Mussolini o el Portugal de Salazar (Moreno, 1993; Moreno, 1999; Sevillano, 2002). El resultado fue una aproximación de carácter histórico que tampoco perduró en el tiempo más allá de las aproximaciones laterales al asunto, realizadas por trabajos sobre las instituciones franquistas en las provincias, que nunca llegaban más allá de los años cincuenta. La nómina de obras de carácter local que hacen referencia a estas elecciones es amplísima (un buen resumen puede encontrarse en Sanz, 2010). No obstante, entre todas ellas cabe destacar por excepcional y por abarcar el periodo completo de la dictadura el trabajo de Martí Marín sobre los Ayuntamientos catalanes (Marín, 2000).

Partiendo de este contexto historiográfico, el presente artículo pretende esbozar las principales líneas a través de las cuales articular una propuesta metodológica y teórica que permita analizar el franquismo desde una perspectiva electoral. Un enfoque que ya ha mostrado sus posibilidades para profundizar en la relación Estado-sociedad y que en el caso español permite además tanto cubrir el periodo menos estudiado del franquismo, es decir, a partir de 1945, como profundizar en el marco comparado de los años 60 y 70, dado que todas las dictaduras europeas posteriores a la II Guerra Mundial organizaron elecciones. Para conseguir este objetivo, a lo largo de las siguientes páginas recurriremos a los trabajos realizados tanto por la ciencia política como por la historia, insertando nuestra investigación en el marco historiográfico citado anteriormente.

\section{Una aproximación pluridisciplinar a un objeto multifacético}

En un famoso artículo publicado en la revista Political Studies en 1967, Richard Rose y Harve Mossawir definieron las elecciones como "the most ubiquitous of contemporary political institutions" (Harve Mossawir and Rose, 1967: 173). Esa infinidad de interconexiones con todas las esferas de la vida política y social, incluso cuando nos referimos a comicios organizados en regímenes no democráticos, convierten a las elecciones en un objeto de estudio extremadamente escurridizo al que, a pesar de ello, todas las disciplinas de las ciencias sociales y humanas se han aproximado. El método de análisis que aquí planteamos trata de combinar las dos disciplinas que, como acabamos de ver, han copado los estudios sobre las elecciones en las dictaduras del siglo XX, la historia y la ciencia política.

Una de las principales virtudes de este enfoque pluridisciplinar es que convierte las elecciones organizadas por el régimen de Franco en una nueva vía de aproximación al estudio de las relaciones entre el Estado y la sociedad, uno de los campos de estudio más fértiles dentro de las ciencias sociales y humanas en los últimos años. No obstante, la lente principal a través de la que nuestra propuesta analiza este asunto es la histórica. Por lo tanto, en las siguientes páginas la descripción, la contextualización y la búsqueda de explicaciones que conecten nuestro objeto de estudio tanto con su tiempo (Guerra Fría) como con el pasado (historia electoral europea), o el interés por profundizar en las actitudes y los 
apoyos sociales al franquismo, permean las herramientas de carácter más funcionalista que ofrece la ciencia política a la hora de ahondar en los mecanismos de cooptación de élites, la articulación de dichas élites para sustentar sus respectivos regímenes o las explicaciones sobre la institucionalización, estabilidad y larga duración de los mismos. En este sentido, nos parece fundamental destacar que la aportación de las ciencias sociales al estudio de este tipo de elecciones permite analizarlas, no solo como mecanismos de cooptación, sino también de creación o adaptación de instituciones que constituyen el centro de la negociación y toma de decisión de dichos regímenes (Putnam, 1976; Geddes, 2006; Gandhi, 2008; Costa, 2009a; Costa, 2009b), puesto que, como señaló Amos Perlmutter, ningún régimen dictatorial puede sobrevivir políticamente sin el apoyo de las élites modernas (Perlmutter, 1981: 11).

Ahora bien, cualquiera que descienda a la realidad electoral del franquismo se verá sepultado por una densa y enrevesada red de elecciones con censos, métodos de votación, ámbitos de influencia, duración de los cargos electos, etc. muy distintos, que llevaron a Miguel Martínez Cuadrado a calificarla como una "inabarcable madeja de insondables complejidades" (Martínez, 1974: 1393 y 1395)2. A pesar de las muchas diferencias que había entre ellas, todas se caracterizan por un elemento común, ninguna ponía en juego los puestos de poder realmente ejecutivo de la dictadura, lo que sirvió a algunos investigadores para catalogarlas como "elecciones no competitivas"(Ruiz de Azúa, 1977: 87; Vanaclocha, 1977; Linz, Hermet and Rouquie, 1978). Carlos Domper ha discutido este concepto y propuesto otro alternativo en (Domper, 2014a).

Aun así, nos parece que lanzarse al análisis de ese heterogéneo conjunto de comicios de forma global no solo implicaría graves dificultades metodológicas, sino que, sobre todo, llevaría a quien se encomendase a tal tarea a empeñar toda su vida académica en ella. Esa es la razón por la cual consideramos que la opción más eficaz de investigar el mundo de las urnas franquistas es reducir el tamaño de la muestra de estudio y analizarla con un enfoque más conceptual y teórico que las inserte dentro de las principales líneas de investigación existentes tanto sobre la dictadura, como sobre las elecciones en este tipo de regímenes.

¿Cómo lograrlo? Nosotros apostamos por el método inductivo, es decir, por seleccionar una muestra representativa del objeto de estudio que nos permita analizar los procesos o elementos en los que estamos interesados, para extraer conclusiones generales a partir de ella. En este sentido, proponemos tres criterios para configurar la muestra de estudio. En primer lugar, prestar atención exclusivamente a aquellos comicios que obligaron a la dictadura a realizar un mayor esfuerzo para controlarlos y, por tanto, a mostrar todos los instrumentos a su alcance para lograrlo. Así pues, parece claro que las candidatas perfectas son las elecciones a concejales municipales por el tercio de representación familiar y las elecciones a procuradores familiares en Cortes por ser las que, con mucha

\footnotetext{
Bajo el franquismo convivieron, además de los dos referenda de 1947 y 1966, siete tipos de elecciones distintas: elecciones a procuradores de la Administración Local; a procuradores sindicales; a procuradores representantes de las corporaciones; a consejeros nacionales representantes de las provincias; a diputados provinciales; a concejales municipales; y a procuradores familiares de representación familiar. Miguel Ángel Ruiz de Azúa (1977) y Miguel Martínez Cuadrado (1974) explicaron brevemente el funcionamiento y características de todas ellas.
} 
diferencia, mayor censo tuvieron ${ }^{3}$. En segundo lugar, limitar la investigación a las capitales de provincia. A pesar de que dejar fuera del campo de estudio los ámbitos rurales limita en cierta medida la representatividad de la muestra, dicha carencia puede suplirse parcialmente incluyendo en ella algunas pequeñas ciudades de carácter rural. Además, esto permite concentrar los esfuerzos investigadores en aquellos lugares donde las luchas por el poder fueron más intensas y respondieron a las tensiones generadas por las propias dinámicas institucionales del régimen, en vez de a personalismos y rencillas familiares. Finalmente, es posible acotar todavía más la investigación seleccionando únicamente las capitales cuyas características permitan cubrir lo más aproximadamente posible los principales cleavages en torno a los cuales se hallaba dividida la sociedad española entre 1945 y 1973, y entre los cuales deberían estar sin duda el agrícola/industrial, el centro/periferia; o el nacionalismo estatal/nacionalismos sub-estatales.

Queda así definido un sujeto histórico de dimensiones razonables cuyo análisis se puede abordar a través de tres perspectivas distintas e interrelacionadas: la descripción formal, el análisis de élites y los apoyos sociales al régimen, y el estudio de las actitudes políticas y el consenso. Todas ellas pueden ser complementadas por la perspectiva comparada, puesto que todos los regímenes dictatoriales europeos de la Guerra Fría organizaron elecciones. Esta combinación permite articular un marco interpretativo complejo y dinámico en el que lo local y lo nacional, lo cuantitativo y lo cualitativo, las miradas desde arriba y desde abajo, se asocian de manera constante, dando a la investigación un carácter mucho más analítico y una mayor capacidad interpretativa sin por ello perder su base empírica.

El primero de los planos a los que debe prestarse atención es el descriptivo. Necesitamos definir claramente las características del objeto de estudio, delimitar sus funciones, alcance y reglas. En definitiva, tenemos que dotarlo de corporeidad y hacerlo aprehensible como sujeto histórico. ¿Quiénes eran los electores?, ¿cuántos eran?, ¿quiénes podían presentarse como candidatos?, ¿cómo se forman las juntas del censo?, ¿y las mesas electorales?, ¿qué sistema de votación se utilizaba, directo o indirecto?, ¿cómo eran las circunscripciones?, ¿qué instituciones se encargaban de la organización de las elecciones?, ¿cómo lo hacían? Todas estas preguntas pueden responderse acudiendo a los textos legales que articularon los comicios que nos interesan. En el caso que nos ocupa los fundamentales son la ley de Bases del Régimen Local de 1945, la ley Orgánica del Estado de 1967, la ley de Representación Familiar en Cortes de ese mismo año y las diferentes órdenes ministeriales con instrucciones para organización de las mismas, todas ellas publicadas en el Boletín Oficial del Estado (en adelante BOE).

No obstante, constituiría un grave error confiar plenamente en la realidad electoral que emerge del $B O E$. En este sentido, Roque Moreno Fonseret subrayó hace algunos años que estos textos legales convivieron durante toda la dictadura con un conjunto de actividades extralegales destinadas a controlar unos comicios ya de por sí muy rígidos y encorsetados. Dichas actividades estuvieron articuladas

Conviene aclarar que los dos referenda organizados por Franco en 1947 y 1966, tuvieron un censo mucho mayor. Sin embargo, quedan fuera de esta propuesta, porque tanto los fines a los que respondían como, a consecuencia de ello, los métodos utilizados para lograrlo fueron muy diferentes. Resumiendo, la cuestión lo máximo posible, cabe decir que si lo que se pretende es analizar el impacto de estas elecciones en el sistema institucional del régimen, entonces dichos referenda no son útiles puesto que ni afectaron a instituciones de la dictadura con poder ejecutivo, ni sirvieron para seleccionar personal político, por lo que no permiten observar mecanismos relacionados con la articulación de las élites políticas. 
por un conjunto de circulares y órdenes reservadas escritas desde la Dirección General de la Administración Local (en adelante DGAL) del Ministerio de la Gobernación y desde la Delegación Nacional de Provincias (en adelante DNP) de FET-JONS(Moreno, 1999). Una circunstancia característica de todos los comicios organizados por regímenes no democráticos.

Esta dicotomía entre la realidad oficial del $B O E$ y la realidad extraoficial de las circulares reservadas, así como la relación profundamente centralizada que existió entre la Administración Central y la Administración Periférica del Estado (Martí, 2000:50-53), permiten profundizar en un campo de estudio prácticamente inexplorado pero de vital importancia para comprender el funcionamiento institucional del franquismo y su larga duración. Nos referimos a los mecanismos que articularon la relación entre la DGAL y la DNP y los gobiernos civiles y las jefaturas provinciales del Movimiento (Sanz, 2010; Marín, 2013). Unos mecanismos que permitieron ejecutar en las provincias las decisiones tomadas por el gobierno central.

¿Tienen estas características alguna conexión con la historia electoral europea? Esta cuestión no es baladí, puesto que, frente a las explicaciones más simples que despachan el tema aludiendo a su carácter meramente legitimador y propagandístico, permite situar la interpretación de los comicios franquistas en un marco mucho más complejo, el de la evolución de los mecanismos electorales y la concesión del derecho al voto en la Europa contemporánea. En ese plano se abre la posibilidad de forjar nuevas herramientas metodológicas para responder cuestiones hasta ahora no ventiladas entre las que nos gustaría destacar las siguientes:

En primer lugar, la inclusión de este tipo de elecciones en el refinamiento del concepto de "participación política" en Europa. Romanelli(1998: 31-32 y 34) apuntó cómo este concepto ha sido desarrollado sin tener en cuenta el análisis de las elecciones que se celebraron en regímenes no democráticos, obviando una parte sustancial de la realidad histórica de la que pretende dar cuenta. Estudios como el que proponemos, al arrojar luz sobre las características y funcionamiento de esas elecciones, muestran cómo las mismas constituyeron mecanismos de participación de una parte de la población en la vida política de esos regímenes no democráticos, bien como electores, bien como candidatos, independientemente del impacto que esa participación tuviese en la toma de decisiones. Esta aportación nos parece importante, porque no parte de la premisa histórica de que los únicos mecanismos de participación de la población en la vida política son aquellos que le permiten influir en la toma de decisiones. De este modo, permite resituar el concepto de "participación política" en una realidad histórica en la que dicha influencia solo se consolidó en Europa Occidental a partir de 1945, con la excepción de España y Portugal, y no fue mayoritaria en Europa hasta 1989.

En segundo lugar, la necesidad de subrayar la continuidad y fuerza del sufragio censitario en la historia electoral europea, lo cual sitúa el sistema electoral franquista en una línea de continuidad histórica que, por supuesto, debería adaptarse al contexto social e histórico en el que tuvo lugar (Bogdanor, 1983). Esta no es tampoco una cuestión menor, ya que el sufragio censitario fue uno de los elementos característicos de los sistemas electorales liberales europeos del siglo XIX y primer tercio del siglo XX (Kahan, 2003; Nohlen y Stöver, 2010). De hecho, la consolidación del sufragio universal masculino y femenino no se produjo en 
Europa occidental hasta 1945, con la excepción de España y Portugal. En la Península Ibérica, la supervivencia de dos regímenes dictatoriales profundamente antidemocráticos, forjados en los años treinta al calor del auge de los fascismos, bloqueó el desarrollo de la democracia liberal y, por tanto, la implementación de uno de sus elementos característicos, el sufragio universal masculino y femenino.

Asimismo, la tarea descriptiva debe dar cuenta de la evolución que todo ese corpus legal al que acabamos de aludir experimentó al calor de la finalización del proceso de institucionalización del régimen y las transformaciones económicas y sociales a las que se vio sometida la sociedad española desde los años sesenta. Una tarea que no solo permite contribuir al debate sobre la supuesta apertura del sistema político franquista y su capacidad, o no, para incluir a nuevos sectores sociales en su seno (un asunto sobre el que es de obligada referencia el libro de Molinero e Ysas, 2008), sino que a través de la comparación con la realidad establecida por la documentación reservada ya comentada permite evaluar la distancia existente entre la apertura teórica de la que hablaban las leyes y la apertura real que permitieron el Ministerio de la Gobernación y el Movimiento. La existencia de esta dualidad no debería pasarse por alto a la hora de analizar los trabajos de algunos intelectuales del periodo sobre la configuración del régimen como un Estado de derecho (sobre el asunto, véase Sesma, 2006).

Pasar de la descripción analítico-formal de estas elecciones a examinar la élite política de segunda fila que accedió a través de ellas a puestos no ejecutivos de instituciones secundarias del régimen, cuyos integrantes estaban completamente subordinados a autoridades superiores designadas de forma discrecional, requiere prestar atención a dos aspectos interrelacionados y conectados con las cuestiones más generales de la institucionalización y los apoyos sociales al franquismo. Por un lado, investigar los mecanismos institucionales que permitieron la selección y elección de los candidatos, así como de los actores responsables de su funcionamiento. Por otro, definir el perfil sociopolítico de dicha élite con el doble objetivo de compararlo con el de aquellas que fueron seleccionadas de forma discrecional, y comprobar en qué grado estuvieron representados en él todos los sectores que sustentaban al régimen. En ambos casos, la prosopografía representa un papel esencial.

¿Cuáles eran los mecanismos que permitían la cooptación de los candidatos y el control de su elección? Para responder a esta pregunta el foco del análisis debe apuntar en dos direcciones, la Administración Central y la Administración Periférica. De hecho, la relación entre ambas debería convertirse en un objeto de estudio preferente, puesto que, sin comprender correctamente la interacción entre las dos, es imposible explicar satisfactoriamente el funcionamiento institucional de la dictadura en provincias. Al margen de los detalles concretos, el gobernador civil de cada provincia era el que se encargaba de configurar las listas de candidatos oficiales y de vigilar, obstaculizar y, en última instancia, anular las posibles candidaturas opositoras. En este sentido, la unificación de los cargos de gobernador civil y jefe provincial del Movimiento a mediados de los cuarenta permitió a las estructuras provinciales del Partido, controladas por la DNP, acceder a los mecanismos de configuración de las candidaturas oficiales (Marín, 2000). Para profundizar en el proceso de implantación de la dictadura en las provincias (véase Marín, 2000; Sanz, 2009; Rodríguez, 2013; León, 2017). 
El análisis prosopográfico de los diferentes gobernadores civiles tiene en este sentido una importancia capital porque permite poner de relieve la militancia falangista de la mayoría de ellos al menos hasta mediados de los sesenta (Sanz, 2010; Marín, 2013). Este es un detalle fundamental para explicar el férreo control que FET logró ejercer sobre estos comicios -especialmente los municipales (Domper, 2013), dado que cuando empezaron a organizarse los de procuradores familiares su poder en provincias se hallaba en declive-, tanto en el proceso de apoyo a los candidatos oficiales (donde su red de prensa tuvo un papel esencial), como en el proceso de selección de los mismos. De este modo, el análisis de estas dinámicas permite analizar el poder real de FET-JONS en la España de Franco después de 1945, un asunto en el que se lleva poco tiempo trabajando (y sobre el que las mejores monografías son, posiblemente: Molinero e Ysas, 2008; Ruiz Carnicer, 2013), y su papel fundamental en la selección del personal político en provincias, al menos hasta la crítica coyuntura de los años sesenta. Por lo demás, estos espacios de investigación abren la puerta a examinar, tanto la articulación de los grupos sustentadores de la dictadura (Schmitter, 1978), como los enfrentamientos que surgieron entre ellos a consecuencia de las dinámicas generadas por ese proceso ${ }^{4}$.

Estos mecanismos no se vieron sustancialmente afectados por los cambios sociales y económicos de los sesenta, aunque, como consecuencia del desplazamiento de FET del control de los gobiernos civiles, sí lo hicieron las relaciones que a través de ellos se habían establecido entre los diversos sectores del régimen. Además, el Movimiento dejó de ser el canal prioritario a través del cual acceder a las candidaturas en un contexto muy permeado por el debate en torno al asociacionismo político y la creciente aparición de candidatos independientes que buscaban plataformas de promoción y que, en ausencia de partidos y asociaciones políticas, acabaron recurriendo en algunos casos a las asociaciones de cabezas de familia. Estos hombres y mujeres, que en absoluto eran opositores, comenzaron a denunciar en los tribunales las irregularidades electorales que la dictadura venía practicando con impunidad desde 1948, lo cual empezó a resquebrajar la eficacia de los mecanismos de control de estas elecciones, al sacar a la luz (al menos judicial) todo el entramado de prácticas extralegales que ya hemos mencionado.

Como consecuencia de todo ello, el análisis de estos mecanismos necesita prestar atención tanto al contenido de las mencionadas reclamaciones, su destino final en los tribunales y la forma en la que condicionaron el desarrollo de las elecciones familiares, como al funcionamiento de las citadas asociaciones y su relación con la construcción de la sociedad civil durante los estertores de la dictadura, un asunto sobre el que contamos con la excelente monografía de la profesora Pamela Radcliff (2011). En este sentido, nos parece fundamental la inclusión en el marco explicativo de los intentos de una parte del falangismo, capitaneada por Solís, de utilizar las elecciones en su estrategia por ampliar la base social de FET y dotar al régimen de una legitimidad alternativa a la de "ejercicio" que venía siendo defendida por los tecnócratas del Opus Dei.

Pero, ¿quiénes eran aquellos candidatos? Resolver esta cuestión implica nuevamente recurrir a la prosopografía a fin de poder trazar perfiles

\footnotetext{
Un claro ejemplo de ello lo encontramos en las elecciones municipales de Madrid de 1954, donde la candidatura integrada por los monárquicos Joaquín Fanjul, Torcuato Luca de Tena, Joaquín Calvo Sotelo y Joaquín Satrústegui denunció irregularidades a favor de la lista falangista, finalmente vencedora.
} 
socioprofesionales y políticos tanto de lo que podríamos llamar el candidato tipo que accedió a estas elecciones como de quienes resultaron finalmente elegidos. En este sentido, los posibles cotejos son múltiples y van desde la comparación entre candidatos y elegidos hasta las más específicas entre candidatos o elegidos por diferentes tercios o tipos de elección pasando por la comparación con los perfiles de élites intermedias o superiores como podrían ser los alcaldes o gobernadores civiles hasta los ministros y demás ocupantes de la alta administración del Estado.

Esta línea de investigación no solo permite contribuir al ya clásico debate sobre la renovación o no del personal político del franquismo en las provincias y, por tanto, al esclarecimiento de la cuestión de los apoyos sociales a la dictadura, sino que ofrece la posibilidad de comprobar si el franquismo permitió a los diferentes sectores que lo apoyaban competir en estas elecciones o si, por el contrario, algunos en concreto fueron los privilegiados. Este enfoque es interesante, porque arroja luz acerca de la representación de dichos sectores en las instituciones y sobre las posibilidades reales de elección que los votantes tuvieron. De este modo, la aproximación de carácter cualitativo mejora el simple análisis cuantitativo hecho muchas veces por los politólogos, que se limitan a dividir el número de puestos en disputa por el número de candidatos con el objetivo de determinar si hubo, o no, "competición".

¿Los cambios que sufrió la sociedad española a partir de los años sesenta se reflejaron en la composición de esta élite de segunda fila? La respuesta a esta pregunta es otro elemento fundamental a tener en cuenta a la hora de afrontar el análisis de los procesos electorales a los que nos estamos refiriendo. En efecto, esta cuestión ofrece la posibilidad de comprobar la medida en que la dictadura fue capaz de incorporar a los nuevos sectores sociales surgidos al calor del desarrollismo y la creación de la élite funcionarial del Estado en las provincias, algo que resulta trascendental para evaluar los límites del aperturismo franquista y sus posibilidades de supervivencia más allá de la muerte de Franco.

En este punto, debemos prestar atención al peso numérico y cualitativo que tuvieron en la conformación de esta élite en el tardofranquismo un conjunto de hombres y mujeres que, sin tener experiencia política previa y sin ser ni mucho menos opositores, comenzaron a presentarse de forma independiente, mayoritariamente a través del conducto familiar, con el objetivo, en muchos casos, de mejorar las cosas en sus ciudades y en las Cortes. Algunos de ellos, además, acabarían teniendo un papel en el proceso de transición a la democracia, como en el caso de Adolfo Suárez, que se presentó como candidato a las elecciones a procuradores familiares en Cortes de 1967 por Ávila. Por lo tanto, no resulta descabellado plantearse la posibilidad de aplicar el concepto de "espacio de experiencia" desarrollado por Koselleck años atrás (Koselleck, 1993).

Por último, nos gustaría poner de manifiesto que los procesos electorales a los que estamos aludiendo permiten también realizar algunas aportaciones a la historia social del franquismo y, especialmente, a uno de los campos de estudio más en boga actualmente dentro de la historiografía sobre la dictadura de Franco, el de las actitudes sociales hacia la misma. Este es un campo de investigación en ebullición cuyos principales debates pueden encontrarse en el magnífico libro de Claudio Hernández (2013). Obviamente, esta aproximación afecta fundamentalmente a los comicios protagonizados por los cabezas de familia, que fueron los que contaron 
con un censo más amplio y heterogéneo. Pensamos que son al menos tres los caminos a transitar en esta dirección. Por un lado, el del estudio de la confección del censo electoral y las características socioeconómicas y políticas de los ciudadanos con derecho a voto en el franquismo. Por otro, el de la articulación de las circunscripciones electorales; y, finalmente, el del análisis de los datos de participación, abstención, votos nulos y en blanco, etc.

Por lo que se refiere a la delimitación de quién tenía derecho a votar en la España de Franco, el camino a seguir viene marcado por los propios textos legales a los que hemos hecho referencia anteriormente. Tanto la Ley de Bases del régimen local como la Ley de Procuradores familiares en Cortes determinan claramente el perfil del votante. Así, recurriendo a documentación oficial, puede fijarse el número de personas censadas (aunque no es fácil encontrar censos electorales del franquismo) y compararse con el total de la población. De este modo, puede calcularse el grado de limitación del sufragio, estableciendo además una conexión directa con una de las características del sufragio en España y Europa durante la edad contemporánea, el carácter censitario del sufragio. El rastreo de esa evolución puede conducirnos de igual manera a observar si con el peso de los años la dictadura amplió el porcentaje de población a la que permitía votar.

Si de la definición de lo que podríamos denominar como cuerpo electoral, pasamos a analizar el comportamiento del mismo de forma conjunta, resulta indispensable determinar el tamaño y composición de las unidades administrativas a través de las cuales se articuló la votación de quienes estaban incluidos en el censo electoral, es decir, la circunscripción electoral. De acuerdo con las órdenes del Ministerio de la Gobernación, era el gobernador civil quien debía fijar esas demarcaciones electorales y darlas a conocer a través de su publicación en el Boletín Oficial de la Provincia. Utilizando la composición de los distritos electorales de cada ciudad aparecidos en esos boletines pueden reconstruirse dichas divisiones sobre un mapa. Aplicando este método, Carlos Domper ha mostrado cómo el franquismo modificó las circunscripciones electorales con el objetivo de evitar que las votaciones reflejasen comportamientos de clase, sobre todo en barrios habitados mayoritariamente por trabajadores industriales (Domper, 2014b).

Esto nos lleva al último de los apartados a los que hemos aludido más arriba. El examen de los datos de las votaciones. Aquí, al igual que en lo referido al tema de los candidatos y los mecanismos para su cooptación, son sin duda de gran utilidad las herramientas desarrolladas por la ciencia política y la sociología electoral. A decir verdad, es sabido que los registros de participación y abstención generales en una dictadura no son elementos de mucha fiabilidad. De hecho, Carlos Domper ha señalado que las cifras oficiales de participación que ofreció el régimen en Zaragoza fueron aumentadas en casi un cincuenta por ciento de forma sistemática (Domper, 2014b).

Sin embargo, junto con los propios informes redactados por los gobernadores civiles, existe una gran diversidad de indicadores que pueden darnos información sobre cuál fue la actitud de los españoles con derecho a voto, en su mayor parte profundamente despolitizados (Hernández, 2013), no solo hacia estos comicios en particular, sino hacia el sistema institucional del franquismo en general. Entre los más relevantes de estos indicadores debemos destacar: la evolución de los porcentajes de participación, su crecimiento significativo asociado a la presencia de 
candidatos no oficiales, el aumento repentino de los votos nulos o/y en blanco, la propia necesidad del Estado de manipular unas cifras de participación en muchas ocasiones realmente bajas, e, incluso, la consiguiente adopción de medidas para estimular la participación a partir de los años sesenta. El análisis electoral del franquismo desde esta perspectiva nos conduce al debate en torno al consenso bajo el régimen franquista, puesto que pone sobre la mesa la cuestión de si dichas elecciones permitieron ampliar o reforzar el consentimiento que existía sobre el régimen o, por el contrario, aquellas fueron usadas como un elemento represivo o coactivo más.

El plano comparado es perfectamente compatible con este tipo de investigación. De hecho, permite poner en relación la dictadura franquista con su entorno geográfico y político, ya que el franquismo no fue la única dictadura que organizó elecciones en Europa tras 1945. Gracias a la historia comparada podemos buscar similitudes y diferencias que nos permiten conocer mejor estas dictaduras. ¿En qué grado concedieron el derecho al voto a sus ciudadanos?, ¿dieron acceso a puestos relevantes en la toma de decisiones?, ¿en qué fase de las dictaduras se pusieron en marcha?, ¿cómo fueron recibidas por quienes debían presentarse a ellas?, ¿y por quienes debían votar?, ¿cuál fue el grado de control e intervención del Estado?, ¿a qué organismos les fue encargada la supervisión y control de los procesos? La respuesta a estas preguntas permite indagar en asuntos relacionados no solo con la institucionalización de dichas dictaduras, sino con su larga duración y con el modo en el que se relacionaron con sus respectivas sociedades en un contexto en el que las elecciones alcanzaron la categoría de elemento simbólico insoslayable en la sociedad occidental.

Por último, abundaremos en dos problemas metodológicos relevantes. En primer lugar, la dificultad que se plantea para analizar dos tipos de elecciones que sirven para cubrir puestos en instituciones diferentes situadas a distintos niveles, el municipal y el nacional, sin caer por ello en lo que Sartori definió como la "falacia del salto unitario" (Sartori, 1970). Esa dualidad complica a priori el análisis combinado de ambas en busca de problemas y dinámicas comunes. Sin embargo, la relación entre ellas se encuentra en su propia mecánica de funcionamiento, puesto que las dos tenían como punto de organización común la provincia. Eran las mismas autoridades las que las organizaban. Además, en el caso de las que congregaban a los cabezas de familia, los candidatos aludían a temas locales y provinciales durante los periodos electorales.

En segundo lugar, es pertinente mencionar los problemas existentes alrededor de la idea de asociar directamente abstención electoral en contextos no democráticos con oposición al régimen. La existencia de esa relación fue en primer lugar teorizada en los años sesenta y setenta por los científicos sociales que analizaron las elecciones que tuvieron lugar en la URSS (Gilison, 1968; Karklins, 1986; Roeder, 1989) y posteriormente aplicada al análisis del caso español por Luis López Guerra (López, 1978). No obstante, pese a los muchos intentos de los politólogos por probar está hipótesis, ningún estudio ha sido definitivo al respecto como señalaron Richter y Jessen (2011: 14). Con todo, antes de descartarla merece la pena tratar de refinarla utilizando el análisis histórico. Es decir, quizás tener en cuenta solo los porcentajes generales de abstención no sea suficiente, pero si comparamos estos porcentajes en zonas muy específicas y descendiendo al nivel de 
los distritos electorales con los resultados obtenidos por partidos de izquierdas en esos mismos distritos en elecciones de carácter democrático anteriores o posteriores, en el caso español elecciones en la II República y primeras elecciones libres tras la muerte de Franco, quizás podríamos ser más precisos.

\section{Fuentes para una aproximación electoral al franquismo}

Las fuentes primarias que permiten dotar de contenido empírico este campo de investigación son abundantes, aunque no exentas de problemas. De hecho, son tanto de carácter nacional como local, lo que permite poner constantemente en contacto ambas esferas. Los principales archivos que custodian la documentación relacionada con este sujeto histórico son el Archivo General de la Administración, especialmente los fondos de Presidencia (donde se encuentra la documentación de la DNP) y Gobernación (donde están los repertorios de la DGAL). El Archivo del Ministerio del Interior, de accesibilidad mucho más restringida, custodia la documentación de la Dirección General de Política Interior, donde puede encontrarse documentación relativa a las elecciones de procuradores de representación familiar en Cortes, una serie que no ha sido trasladada al AGA. Por su parte, en la Biblioteca Nacional, pueden consultarse los principales periódicos de tirada nacional del Régimen, como Arriba o YA. A nivel local/provincial resultan de interés los archivos municipales, los históricos provinciales y los de las subdelegaciones provinciales del gobierno, donde se conservan (si no han sido destruidos) los fondos de los antiguos gobiernos civiles. Finalmente, resultan de interés las encuestas realizadas por el Instituto de Opinión Publica desde los años sesenta, no publicadas en muchos casos, que actualmente pueden ser consultadas en el Centro de Investigaciones Sociológicas.

La accesibilidad de estas fuentes es amplia aunque deben señalarse dos importantes excepciones. Por un lado, la documentación oficial relativa a las elecciones de procuradores familiares en las Cortes no se encuentra, como acabamos de decir, ni en el Archivo General de la Administración ni en el Archivo del Congreso de los Diputados, sino en el Archivo del Ministerio del Interior, lo que dificulta su consulta, puesto que el acceso depende de la decisión discrecional de los gestores de dicho archivo. La segunda de las excepciones afecta a las fuentes de ambos comicios, municipales y procuradores familiares en Cortes, y tiene que ver con las restricciones de acceso a documentos con información personal de los candidatos a partir de la década de los sesenta, lo que complica el análisis de las elecciones a procuradores familiares en Cortes y dificulta el de las elecciones locales a partir de esa fecha.

Para realizar el análisis descriptivo y de la propia mecánica electoral las principales fuentes documentales a tener en cuenta son tanto el $B O E$ como los boletines oficiales de las respectivas provincias, donde se puede encontrar toda la legislación relativa a estas elecciones. En este sentido, también resultan claves las circulares e instrucciones emitidas por la DGAL y la DNP, así como la correspondencia entre los gobernadores civiles y ambas instituciones nacionales, gracias a la cual es posible documentar todas las prácticas extralegales que 
acompañaron y subvirtieron el teórico orden legal definido por las publicaciones del $B O E$ y las circulares regulares.

La documentación disponible para investigar la élite política de segunda fila que surgió de estas elecciones es más amplia. Entre los documentos de mayor interés están los estadillos de información personal de los candidatos que fueron confeccionados por la DNP, hasta mediados de los sesenta, y por la DGAL, a partir de entonces, utilizando los informes que enviaban los jefes provinciales del Movimiento, primero, y los gobernadores civiles, después. Esta fuente es especialmente interesante para las elecciones por el tercio familiar y sobre todo en lo que hace referencia a los candidatos que resultaron finalmente elegidos. No obstante, en los archivos de los gobiernos civiles que se conservan en algunas provincias puede encontrarse información sobre los candidatos que no fueron elegidos. También son de interés los informes realizados por los jefes provinciales del Movimiento, a petición de la DNP tras las elecciones de 1954, y los gobernadores civiles con ocasión del referéndum y las elecciones locales que tuvieron lugar en 1966.

Por desgracia, como consecuencia de las razones ya señaladas, la accesibilidad a estos repertorios de primera mano acerca de las elecciones a procuradores familiares en las Cortes es mucho más limitada. Por tanto, la prensa, que es un muy buen instrumento para complementar la información sobre las elecciones locales, se convierte en una fuente prioritaria para el análisis de estos otros procesos electorales. Junto a ella, las entrevistas a los protagonistas constituyen una de las principales herramientas que pueden ayudarnos a superar la ausencia de documentación oficial, ya que las elecciones a procuradores familiares en Cortes tuvieron lugar en 1967 y 1971 y a ellas se presentaron muchos candidatos relativamente jóvenes, sobre todo a las primeras, que siguen vivos a día de hoy. Finalmente, en el archivo del Congreso de los Diputados podemos encontrar información sobre algunos de los que finalmente fueron elegidos, utilizando las credenciales de los procuradores que allí se conservan.

Documentar el estudio "desde abajo" de los procesos electorales franquistas es una tarea más complicada por dos razones. En primer lugar, porque las fuentes de las que disponemos para hacerlo son de menor cuantía que las anteriormente citadas. En segundo lugar, porque las mismas nos aportan una visión indirecta del modo en el que los ciudadanos afrontaron estas elecciones. Por un lado, lo hacen a través de los ojos de las autoridades que relataron los informes. Por otro, a través de la interpretación que el investigador hace de las cifras de participación, abstención, votos nulos o en blanco, etc.

Por lo que se refiere al estudio del sufragio y su extensión a lo largo del periodo analizado, la mejor herramienta de la que disponemos, aunque no se han conservado en todas las provincias, son sin duda los censos electorales. La información que estos aportan puede complementarse con la existente en los informes sobre las diferentes elecciones realizados por los gobernadores civiles y los jefes provinciales del Movimiento, donde aparecen también el número de delegados que votaron en las elecciones por el tercio sindical. La prensa constituye otra fuente para conocer el número de personas censadas para cada elección por el tercio de representación familiar, tanto a nivel local como de procuradores en Cortes desde 1967. 
En otro orden de cosas, la reconstrucción de los distritos electorales es posible gracias a los boletines oficiales de la provincia y la prensa. En ambos se publicaban las calles que componían cada distrito electoral para que cada cabeza de familia supiese cual era el centro al que tenía que ir a depositar el voto. Por otro lado, los datos sobre participación, número de votos, abstenciones, etc. pueden encontrarse tanto en los previamente citados estadillos realizados por la DNP y la DGAL, como en la prensa, que en el caso de los periódicos locales da cuenta en muchos casos de la distribución de los votos por distritos.

Finalmente, la opinión de los ciudadanos sobre estas elecciones puede analizarse de manera indirecta a través de los informes que los gobernadores civiles y jefes provinciales del Movimiento realizaban sobre las elecciones de cada provincia, los comentarios que los primeros hacían al respecto en los informes anuales de actividades de los gobiernos civiles y los segundos en los partes mensuales que hasta principios de los cincuenta enviaban a la DNP. Desde la perspectiva de la oposición, al menos de una parte de ella, son relevantes los diversos informes que escribieron miembros del Partido Comunista en la clandestinidad y que pueden consultarse en el Archivo Histórico del Partido Comunista de España. Asimismo, una última vía de análisis sería la proporcionada por las encuestas electorales que realizó el Instituto de Opinión Publica desde los años sesenta referidas a elecciones locales y a elecciones a procuradores familiares.

\section{Algunos retos en torno al estudio de las elecciones, el franquismo y la Guerra Fría}

Nos gustaría terminar poniendo de relieve algunos de los retos que plantea para el historiador el análisis del franquismo desde una perspectiva electoral. Aun cuando son muy variados, queremos subrayar aquellos relacionados con lo que consideramos una tarea fundamental de la historiografía actual del franquismo, la necesidad de integrar las conclusiones de las investigaciones realizadas en el marco de debates conceptuales e historiográficos más amplios relacionados con la inclusión de la historia de la dictadura de Franco en el marco de la historia contemporánea de Europa, en general, y de la Guerra Fría en particular.

Cuando Mark Mazower publicó su famosa obra Dark Continent en 1998 (Mazower, 1998), puso de manifiesto cómo la historia de las dictaduras europeas posteriores a la II Guerra Mundial había sido excluida de la construcción de la historia de Europa occidental tras 1945, una idea que vuelve a aparecer en la introducción del no menos reconocido Postwar de Tony Judt (Judt, 2007). En este sentido, nos parece que integrar la historia de las dictaduras europeas en ese marco debería de ser una de los principales retos de quienes las estudiamos. El hecho de que todas ellas realizaran elecciones no es en absoluto baladí, puesto que constituye no solo una línea de conexión entre las mismas, sino también con el propio contexto histórico de la Guerra Fría en el que tuvieron lugar y ello por dos razones. En primer lugar, porque el hecho electoral se convirtió, tras la firma del Tratado de Yalta, en un elemento simbólico inevitable para cualquier régimen que quisiera integrase en el Orden Internacional surgido de las cenizas de la guerra. En segundo lugar, debido a que, al margen de las manidas y nunca suficientemente 
probadas funciones legitimadoras de dichos procesos electorales, parece que todos ellos contribuyeron a la institucionalización de aquellas dictaduras en la convulsa segunda mitad de los años cuarenta.

En esta misma línea, y conectando con el hecho subrayado durante la introducción de que los estudios de la dictadura de Franco siguen sufriendo un gran desequilibro a favor de los que se dedican a los años anteriores a la década de los cincuenta, la investigación de estas elecciones ofrece una escenario perfecto no solo para continuar aportando luz sobre el periodo menos transitado por la historiografía, sino también para plantear conceptualizaciones distintas del régimen que nos permitan comprender mejor su naturaleza política tras la derrota de los fascismos. En este sentido, nos parece de lo más sugerente intentar integrar la celebración de estas elecciones y sus principales características dentro del proceso evolutivo que la síntesis fascista surgida de la guerra civil (Gallego 2014) experimentó a partir de los años cincuenta, lo que le llevó a transformarse de acuerdo con las imposiciones de un contexto de Guerra Fría completamente distinto al que dio origen al régimen. En este sentido, la aplicación del concepto de "postfascismo" al régimen de Franco, en el sentido que lo están haciendo Miguel Ángel Ruiz Carnicer y su grupo de investigación, constituye el marco teórico perfecto para realizar esa integración (Ruiz Carnicer, 2014).

Finalmente, otro de los retos que debería afrontar cualquier investigación del franquismo desde la perspectiva electoral tiene que ver con la retrospectiva. En general, el estudio de los procesos electorales en las dictaduras ha tendido a realizarse aceptando el marco teórico inicial desarrollado por los científicos sociales. Es decir, para discernir entre unas elecciones dignas de tal nombre y que puedan ser calificadas de democráticas y aquellas que constituyen una mera fachada propagandística sin mayor función que la de lograr legitimidad, sobre todo internacional, el factor esencial recae en si dichos procesos electorales son competitivos o no. Sin embargo, este marco teórico separa por completo el objeto de estudio del marco histórico, convirtiéndolo en un hecho contingente solo sujeto a un análisis funcionalista. El objetivo, por lo tanto, consiste en situar estos comicios en el marco más amplio de la historia electoral europea. Tratar de entender estas elecciones en el contexto de la evolución de los sistemas electorales europeos desde el siglo XIX y observar cuáles son sus conexiones y rupturas con ellos, porque parece evidente que el sufragio censitario y la escasa representación de los sectores sociales entre los candidatos que se presentaban a las elecciones no fue algo exclusivo del franquismo y el resto de dictaduras europeas posteriores a la segunda guerra mundial, sino que fue moneda de cambio habitual en la historia europea del XIX.

\section{Bibliografía}

Altman, D. (2011): "Competition, Political”, en B. Badie, D. Berg-Schlosser y L. Morlino, eds., International Encyclopedia of Political Science, Thousand Oaks, SAGE Publications, pp. 360-364.

http://dx.doi.org/10.4135/9781412959636.n87 
Bogdanor, V., ed., (1987): Democracy and elections: electoral systems and their political consequences, Cambridge, Cambridge University Press.

Chrystal, W. G. (1975): "Nazi party election films”, Cinema Journal, 15, pp. 29-47.

Costa, A. (2009a): "Eleiçoes e ditaduras. Um paradoxo estável-comentario", en A. Freire, ed., Eleiçoes e sistemas eleitorais no século XX portugués: uma perspectiva histórica e comparativa, Lisboa, Ediçoes Colibri, pp. 209-212.

Costa, A., ed., (2009b): Ruling elites and decision-making in fascist era dictatorships, New York, Columbia University Press.

Dal Lago, P. (1999): Verso il regime totalitario: i plebiscito fascista del 1929, Padova, Cleup.

Domper, C. (2013): "Entre la fuerza del mastodonte y la reserva de dinosaurios. Falange y las elecciones municipales de representación familiar en Aragón, 1948-1973”, en M. A. Ruiz, ed., Falange. Las culturas políticas del fascimo en la España de Franco, Zaragoza, Institución 'Fernando el Católico', pp. 111-130

Domper, C. (2014a): "La otra cara de las urnas. Elecciones, dictaduras y Guerra Fría en Europa. Propuesta para un marco conceptual", Rúbrica Contemporánea, 5, pp. 45-69. http://www.raco.cat/index.php/rubrica/article/viewFile/286263/374377

Domper, C. (2014b): "Las elecciones de Franco en Zaragoza (1948-1973). Una aproximación local a un asunto transnacional", Ayer: Revista de Historia Contemporánea, 94, pp. 201-228.

Fimiani, E. (1997): "La legittimazione plebiscitaria nel fascismo e nel nazionalsocialismo. Una interpretazione comparata", Quaderni Storici, 94, pp. 183-224.

Friedgut, T. H. (1979) Political participation in the USSR, New Jersey, Princeton University Press.

Gallego, F. (2014): El evangelio fascista. La formación de la cultura política del franquismo (1930-1950), Barcelona, Crítica.

Gandhi, J. (2008): Political institutions under dictatorship, Cambridge, Cambridge University Press.

Geddes, B. (2006): "Stages of development in Authoritarian Regimes" en V. Tismaneu, M. M. Howard y R. Sil, eds., World order after Leninism, Seattle y London, The University of Washington Press, pp. 149-170.

Gilison, J. M. (1968): "Soviet Elections as a Measure of Dissent: The Missing One Percent", The American Political Science Review, 62, pp. 814-826. http://dx.doi.org/10.2307/1953432

Harve M. y R. Rose (1967): "Voting and elections: a functional analisys", Political Studies, 15(2), pp. 173-201 . http://dx.doi.org/10.1111/j.1467-9248.1967.tb01843.x

Hernández, C. (2013): Franquismo a ras de suelo. Zonas grises, apoyos sociales y actitudes durante la dictadura, 1936-1976, Granada, Universidad de Granada.

Jessen, R. y H. Richter (2011): Voting for Hitler and Stalin. Elections under 20th Century Dictatorships, Frankfurt, Campus Verlag.

Judt, T. (2007): Postwar: a history of Europe since 1945, London, Pimlico.

Jung, O. (1995): Plebiszit und Diktatur: die Volksabstimmungen der Nationalsozialisten. Die Fälle 'Austritt aus dem Völkerbund' (1933), 'Staatsoberhaupt' (1934) und 'AnschluB Österreichs' (1938), Tubingen, Mohr Siebeck.

Kahan, A.S. (2003): Liberalism in Nineteenth-Century Europe. The Political Culture of Limited Suffrage, New York, Macmillan Palgrave. 
Karklins, R. (1986): "Soviet Elections Revisited: Voter Abstention in Noncompetitive Voting", The American Political Science Review, 80, pp. 449-470 . http://dx.doi.org/10.2307/1958268

Koselleck, R. (1993): “'Espacio de experiencia' y 'Horizonte de expectativa', dos categorías históricas" en R. Koselleck, ed., Futuro pasado. Para una semántica de los tiempos históricos, Buenos Aires, Paidós, pp. 333-359.

León, A. (2017): La retaguardia de Franco. Personal politico y poder local en las Canarias Occidentales 1935-1961, La Laguna, Instituto de Estudios Canarios.

López, L. (1978): “Abstencionismo electoral en contextos no democráticos y de transición: el caso español”, Revista Española de Investigaciones Sociológicas, 2, pp. 53-69. http://www.jstor.org/stable/40182693?seq=1\#page_scan_tab_contents

Linz, J. J., G. Hermet y A. Rouquie (1978): Des élections pas comme les autres, Paris, Foundatine Nationale de Sciences Politiques.

Marín, M. (2000), Els ajuntaments franquistes a Catalunya: politica $i$ administració municipal, 1938-1979, Lleida, Pages.

Marín, M. (2013): “Los gobernadores civiles del franquismo, 1936-1963", Historia y Política, 29, pp. 269-299.

http://www.cepc.gob.es/eu/argitalpenak/aldizkariak/aldizkarielektronikoak?

$\mathrm{IDR}=9 \& \mathrm{IDN}=1310 \& \mathrm{IDA}=36744$

Martínez, M. (1974): “Representación. Elecciones. Referéndum” en M. Fraga, J. Velarde y S. Del Campo, eds., La España de los años 70. Vol.3, Madrid, Moneda y Crédito, pp. 1371-1439.

Mazower, M. (1998): Dark Continent. Europe's Twentieth Century, London, The Penguin Press.

Molinero, C. y P. Ysàs (2008): La anatomía del franquismo. De la agonia a la supervivencia, 1945-1977, Barcelona, Crítica.

Moradiellos, E. (2003): "Propuestas de conceptualización política e histórica", en E. Moradiellos, La España de Franco (1939-1975). Política y sociedad, Madrid, Síntesis, pp. 11-23.

Moreno, R. (1993): “La presencia de los grupos políticos en el régimen de Franco a través de las elecciones municipales de 1948" en J. Tusell, J. Gil y F. Montero, eds., Estudios sobre la derecha española contemporánea, Madrid, UNED, pp. 613-626.

Moreno, R. (1999): "Las consultas populares franquistas: la ficción plebiscitaria" en R. Moreno y F. Sevillano, eds., El franquismo. Visiones y balances, Alicante, Prensas de la Universidad de Alicante, pp. 77-175.

http://publicaciones.ua.es/filespubli/pdf/LD84790850889502494.pdf

Morse, Y. L. (2012): “The era of electoral authoritarianism", World Politics, 64, pp. 161-198. http://dx.doi.org/10.1017/S0043887111000281

Mühlen, P. (1979): Schlagt Hitler an der Saar!: Abstimmungskampf Emigration u. Widerstand in Saargebiet 1933-1935, Boon, Verlag neue Gesellschaft.

Nohlen, D. y P. Stover (2010): Elections in Europe: A Data Handbook, Baden-Baden, Nomos.

Ortega, T. M. (2006): "Se hace camino al andar. Balance historiográfico y nuevas propuestas de investigación sobre la dictadura franquista", Ayer: Revista de Historia Contemporánea, 63, pp. 259-278. 
Payne, S. G. (1961): Falange. A History of Spanish Fascism, Stanford, Stanford University Press.

Pérez, J. M. (1994): "Una Dictadura por la gracia de Dios", Historia Social, 20, pp. 173-193.

Perlmutter, A. (1981): Modern authoritarianism. A comparative institutional analysis, New Haven, Yale University Press.

Putnam, R. (1976): The comparative study of political elites, Eaglewood Cliffs, Prentice Hall.

Radcliff, P. (2011): Making Democratic Citizens in Spain. Civil Society and the Popular Origins of the Transition, 1960-78, New York, Palgrave Macmillan.

Rodrigo, J. (2009): "La naturaleza del franquismo: un acercamiento desde la perspectiva comparada de los fascismos europeos", en C. Romero y A. Sabio Alcutén, eds, Universo de Micromundos, Zaragoza, Institución "Fernando el Católico", pp. 47-64.

Rodríguez, Ó. (2006): "La histórica local y social del franquismo en la democracia, 1965-2003. Datos para una reflexión”, Historia Social, 56, pp. 153-175.

Rodriguez. Ó. (2013): Miserias del poder: los poderes locales y el nuevo Estado franquista 1936-19651, Valencia, Publicaciones de la Universitat de València.

Roeder, P. G. (1989): “Electoral Avoidance in the Soviet Union”, Soviet Studies, 41, pp. 462-483. http://dx.doi.org/10.1080/09668138908411826

Roemer, J. (200): Political competition: Theory and applications, Cambridge, Harvard University Press.

Romanelli, R. (1998): "Electoral systems and social structures" en R. Romanelli, ed., How did they become voters? The history of franchise in Modern European Representation, La Haya, Kluwer Law International, pp. 1-36.

Ruiz Carnicer, M. Á., ed., (2013): Falange. Las culturas políticas del fascismo en la España de Franco 1936-1975, Zaragoza, Institución "Fernando el Católico". http://ifc.dpz.es/publicaciones/ver/id/3279

Ruiz Carnicer, M. Á. (2014): "Fascismo, posfascismo y transición a la democracia. La evolución política y cultural del franquismo en relación al modelo italiano", Itinerari di ricerca storica, 1 , pp. 67-88. http://siba-ese.unisalento.it/index.php/itinerari/article/view/14430

Ruiz de Azúa, M. Á. (1977): "Las elecciones franquistas (1942-1976)”, Historia 16, Abril, pp. 80-95.

Sanz, J. (2009): La construccion de la dictadura franquista en Cantabria. Instituciones, personal político y apoyos sociales (1937-1951), Santander, Universidad de Cantabria.

Sanz, J. (2010): "Jerarcas, caciques y otros camaradas. El estudio de los poderes locales en el primer franquismo", Historia del Presente, 15, pp. 9-26.

Sanz, J. (2013): "Falangismo y dictadura. Una revisión de la historiografía sobre el fascismo español" en M. A. Ruiz, ed., Falange. Las culturas politicas del fascismo en la España de Franco, 1936-1975, Zaragoza, Institución 'Fernando el Católico', pp. 25-60. http://ifc.dpz.es/recursos/publicaciones/32/79/03sanzhoya.pdf

Sartori, G. (1970): "Concept Misformation in Comparative Politics", American Political Science Review, LXIV(4), pp. 1033-1053.

http://dx.doi.org/10.2307/1958356 
Sartori, G. (1976): Parties and Party system. A framework for analysis, Cambridge, Cambridge University Press.

Saz, I. (2004): "Algunas consideraciones a propósito del debate sobre la naturaleza del franquismo y el lugar histórico de la dictadura" en I. Saz, Fascismo y franquismo, Valencia, Universitat de València, pp. 245-264.

Schedler, A., ed., (2006): Electoral authoritarianism: the dynamics of unfree competition, Boulder, Lynne Rienner Publishers.

Schmitter, P. C. (1978): "The impact and meaning of 'non-competitive, non-free and insignificant' elections in Authoritarian Portugal, 1933-1974" en G. Hermet, A. Rouquie y R. Rose, eds., Elections without choice, London, Macmillan, pp. 145-168.

Sesma, N. (2006): "Franquismo, ¿Estado de derecho? Notas sobre la renovación del lenguaje de la dictadura durante los años sesenta", Pasado y memoria: Revista de historia contemporánea, 5, pp. 45-58.

https://rua.ua.es/dspace/bitstream/10045/5917/1/PYM_05_03.pdf

Sevillano, F. (2002): "El nuevo Estado y la ilusión de la 'democracia orgánica'. El referéndum de 1947 y las elecciones municipales de 1948 en España”, Historia Contemporánea, 24, pp. 355-387.

http://www.ehu.eus/ojs/index.php/HC/article/view/5978/5658

Swearer, H. R. (1961): "The Functions of Soviet Local Elections", Midwest Journal of Political Science, 5, pp. 129-149.

http://dx.doi.org/10.2307/2109266

Thomàs, J. M. (2008): "Los estudios sobre las Falanges (FE de las JONS y FET de las JONS): revisión historiográfica y perspectivas", Ayer: Revista de Historia Contemporánea, 71, pp. 293-318.

Vanaclocha, F. J. (1977): "Las elecciones de representación familiar en las Cortes españolas (1967-1971) como elecciones no competitivas", Cuadernos Económicos del ICE, 1, pp. 59-76. 